\section{Análisis Estratigráfico del cuerpo de torres de la Catedral Vieja de Salamanca}

José ÁNGel LeCANDA EstebAn

Cata XXI S.L.

\begin{abstract}
Resumen
Las labores arqueológicas desarrolladas en el Cuerpo de Torres de la Catedral Vieja de Salamanca con motivo de su restauración, básicamente y en su primera fase, han consistido en la documentación arqueológica y lectura estratigráfica de sus paramentos, en la toma de muestras para analíticas, así como, paralelamente, en la realización de un nuevo estudio históricodocumental y en el levantamiento de diversas planimetrías. Sin embargo ello no ha sido óbice para profundizar, desde esta primera fase, en el conocimiento histórico de sus etapas constructivas. El empleo de la arqueología aplicada a la restauración, en éste caso, no fue planificado desde el comienzo, como una técnica complementaria necesaria, si no como instrumento de resolución de teorías - y acciones - restauradoras contrapuestas.
\end{abstract}

Palabras Clave: Catedral de Salamanca; Lectura Estratigráfica; Documentación Arqueológica; Analíticas; Etapas Constructivas

\footnotetext{
Abstract

The archaeological work carried out in the body of towers at the Catedral Vieja in Salamanca as part of their restoration, has in the first phase consisted basically of archaeological documentation and stratigraphic readings of its facings, taking samples for analysis, in addition to a new historical-documentary study and various surveys. However, this has not been an obstacle to deepening, from the first phase, historical knowledge of the cathedral's construction stages.

The use of applied archaeology in restoration, in this case, was not planned from the start as a necessary complementary technique, but rather as an instrument for resolving opposed restoration theories and practices.
}

Key words: Salamanca Cathedral; Stratigraphic Reading;

Archaeological Documentary; Analyses; Construction stages.

\section{INTRODUCCIÓN}

Cuando a comienzos del año 2000 empezamos con las labores de análisis estratigráfico del Cuerpo de Torres de la Catedral Vieja de Salamanca, los trabajos de restauración de la misma ya habían comenzado tiempo atrás; ahora se acometía, siguiendo las indicaciones y planificación del Plan Director - elaborado y dirigido por el arquitecto D.Valentín de Berriochoa (1997-98 y 1998)—, una nueva fase de trabajo en el templo (BERRIOCHOA, 1999) y, en ésta, las diferencias de opinión respecto al valor e interés de algunos paramentos y elementos arquitectónicos, tema que «enfrentaba» a técnicos y administración, motivaron nuestra incorporación al equipo, financiada por la Dirección General de Patrimonio de la Junta de Castilla y León y aceptada, en última instancia, por todas las partes implicadas.

Otra vez se recurría a la arqueología como medio puntual de resolución entre ideas y teóricas contrapuestas en el terreno de la restauración monumental, y no de forma previa, intencionada y como parte de un conjunto de ciencias, técnicas y disciplinas necesarias y habituales para este tipo de actuación en elementos del patrimonio histórico. Esta parte introductoria de nuestra comunicación empieza a ser repetitiva en nuestra bibliografía (LECANDA, 1998 y 2000); posiblemente porque todavía falta una verdadera concienciación en el mundo de la restauración sobre la necesidad y utilidad que nuestra ciencia tiene para este tipo de trabajo, o porque se corresponde con el escaso compromiso social que, inconscientemente (suponemos), muestran muchos de los agentes que intervienen en un proceso directo, físico, sobre bienes públicos. Y ello pese a que los fundamentos legales, alegatos lógicos, razonamientos técnicos y ejemplificaciones de sus virtudes no faltan en la bibliografía (especialmente Brogiolo, 1995; Latorre y Caballero, 1995 y PARENTI, 1995).

Resulta obvio señalar que la dificultad para introducir en los equipos y proyectos de restauración la arqueología de la arquitectura es inversamente proporcional al grado de envergadura, monumentalidad o simbolismo del elemento a tratar.

Pues bien, en este contexto, eso sí, envuelto de buenas voluntades por todas las partes involucradas, debe enmarcarse el trabajo de análisis estratigráfico que realizamos en la Catedral Vieja de Salamanca; al respecto, nos parecen bastante significativas algunas cuestiones. Por ejemplo, el objeto de estudio era solo «el cuerpo de torres de la Catedral Vieja, desde la planta baja hasta la cota de la Sala de Bóvedass o, en este mismo sentido, que la lectura de los paramentos debiera producirse en una parte del edificio donde aunque se habían paralizado las obras ya había sido parcialmente alterada por demoliciones, repicados, hormigonado de suelos o lla- 
gueados de paredes, y, finalmente, que para el área de intervención ya existiera un proyecto aprobado de rehabilitación.

Se nos solicitaba que evaluáramos arqueológicamente esta zona del edificio para determinar la importancia histórica de cada una de sus partes, en virtud, sobre todo, de su adscripción a una u otra fase constructiva.

También existía cierto escepticismo entre algunos agentes locales, vinculados a escuelas y metodologías obsoletas pero bien arraigadas y convencidas de que los estudios artísticos e históricos ya existentes sobre el edificio arrojaban toda la luz necesaria y posible sobre tan emblemático monumento, aunque en sus páginas se adivinan lagunas, contradicciones y dudas que, paradójicamente, ellos mismos han señalado.

Sin embargo, algunas de esas aportaciones han sido importantes, sobre todo para permitir una aproximación al edificio y para argumentar una línea interpretativa de los resultados. Destacan, en este sentido, algunas obras fundamentales y que, cronológicamente ordenadas, son las de Julio González (1943), Camón Aznar (1958), Portal Monge (1992 y 1997) y, finalmente, Berriochoa (1997-98). Los resultados, inéditos, de las excavaciones realizadas en la seo con anterioridad (CABALLERO y RETUERCE, 1998) nos permitían tener información sólida sobre la secuencia cultural de la ocupación del lugar, y la bibliografía metodológica (compendiada en INFORMES, 1995 y ACTAS, 1996) nos aportaba el utillaje necesario para comenzar nuestro trabajo.

\section{OBJETIVOS}

Como hemos señalado líneas más arriba, el objetivo oficial, contractual, de nuestro trabajo era la lectura estratigráfica, con su correspondiente documentación arqueológica, del Cuerpo de Torres de la Catedral Vieja de Salamanca.

Inicialmente se acometería la toma de datos, posteriormente se realizarían las oportunas analíticas y, finalmente, se procedería a una síntesis interpretativa que explicara el devenir histórico de tan importante templo señalando fases y etapas, determinando cronologías...

Con ello se perseguía, de un lado, ayudar a orientar las directrices restauradoras del arquitecto (arqueología aplicada) y, aprovechando esa oportunidad, avanzar en la resolución de la problemática histórica que envuelve al edificio (arqueología método histórico).

Para el primer caso, las preguntas son del tipo ¿Tuvo Torre Mocha terraza o cubierta? ¿Por qué se abrieron varias escaleras y husillos en la misma torre? ¿Los diferentes materiales pétreos empleados en el husillo cuadrangular a qué responden? ¿Son todos del mismo interés histórico?, ¿El debilitamiento estructural de las torres es producto del terremoto de Lisboa?, etc.
Para el segundo, las cuestiones a resolver son otras ¿¿Son tres (GONZÁlEZ) o cinco (BERRIOCHOA) las etapas constructivas? ¿Se levantó el templo románico sobre la antigua sede episcopal visigoda de la que nos hablan las fuentes? ¿Fue Torre Mocha una construcción militar preexistente? ¿La semicolumna del acceso viejo es prerrománica?, etc.

Lamentablemente, ahora, no podremos avanzar en el conocimiento histórico, tema que por nuestra parte queda pendiente de acometer y sobre el que vamos avanzando con informaciones muy interesantes, como las relativas a las cronologías.

\section{METODOLOGÍA}

\section{El equipo}

Para acometer las tareas explícitamente señaladas o para coadyuvar a las que implícitamente corresponden al análisis arqueológico, se conformó el siguiente equipo:

—Levantamiento Planimétrico: Fernando Inés Gallo y Félix Zarzuelo, arquitectos.

—Fotogrametría: Ct3 Servicios de Ingeniería Auxiliar.

-Documentación Histórica: Dr. D. Iñaki Martín

Viso, Univ. Salamanca.

—Análisis Petrológico: Dr. D. Mario García Bartual, Univ. Complutense.

-Metalografía: Drs. D. Antonio José Criado y D. Juan

Antonio Martínez, Univ. Complutense de Madrid.

—Sondeo Arqueológico: Aratikos Arqueólogos S.L.

\section{El ámbito de estudio}

La zona a estudiar comprendía parte del Cuerpo de Torres; concretamente, Torre Mocha entera, desde planta baja hasta la terraza, Torre de Campanas, desde planta baja o Capilla de San Martín hasta la Sala de Bóvedas o tercera planta y, finalmente el muro hastial de la nave de la Epístola. Es decir, un gran volumen edificatorio, tanto en planta como en alzado, muy compartimentado e intercomunicado con escaleras, husillos, pasillos, terrazas, etc., con forros distintos en sus paramentos internos y externos (a todas luces de distinta época, pero muy molestos para poder rastrear las continuidades en el volumen edificado de cada unidad estratigráfica muraria) y, para terminar de complicarlo todo, con varios paramentos ya repicados y rejuntados, algunos muros tirados, suelos restaurados....

A fin de establecer una estrategia operativa se procedió a determinar unidades de trabajo, equivalentes a los tradicionales sectores de excavación; éstos se fijaron en virtud de homogeneidad funcional y arquitectónica, y se denominaron ambientes. 
Tienen, además, otra característica y es que entre uno y otro siempre hay un nexo físico, un punto de relación estratigráfica que posteriormente nos permita montar la secuencia relativa de la edificación. Se individualizaron doce ambientes, que son los siguientes (las denominaciones se corresponden con las tradicionalmente empleadas y no implican, necesariamente, relación funcional exacta): Mazmo- rra, Acceso Viejo, Escalera Vieja (Tramo Mazmorra-Carcelero), Escalera Vieja (Tramo Carcelero-Alcaide), Sala del Carcelero, Escalera de Tramos Rectos, Sala del Alcaide, Husillo Restaurado, Husillo Cegado, Terraza, Capilla de San Martín y, finalmente, Nave de la Epístola.

En una distribución por plantas y torres, tendríamos el siguiente cuadro:

\begin{tabular}{clll}
\hline Planta & Torre campanas & Sobre-pórtico & \multicolumn{1}{c}{ Torre mocha } \\
\hline 2 & Husillo Cegado & Sala del Alcaide & Terraza \\
$1 / 2$ & Husillo Cegado? & & Esc. Vieja (C-A) \\
& & Husillo Restaurado \\
1 & Husillo Cegado? & Sala Carcelero & Nave Epístola \\
$0 / 1$ & Husillo Cegado? & Esc. Tramos Rectos & Esc. Vieja (M-C) \\
\hline
\end{tabular}

(Véase Sección en la figura 1)

\section{Aspectos Metodológicos}

Pese a ser una decisión técnico-administrativa nuestra incorporación al equipo, y pese a pretenderse una intervención seria y operativa, los medios destinados tal efecto distaron mucho de corresponderse con las pretensiones enunciadas.

Así, deben buscarse alternativas a las técnicas y protocolos propuestos por los pioneros en esta disciplina; deben simplificarse los procedimientos expuestos en la arqueografía al uso. En nuestra opinión, creemos que en la Catedral Vieja se ha logrado esa mixtura de posibilismo capaz de ofrecer resultados satisfactorios, eso sí, para los fines taxativamente señalados: la lectura estratigráfica y la toma de datos. Por eso su interpretación en clave histórica queda pendiente.

Los fundamentos metodológicos no varían; a saber:

- Los edificios son estructuras orgánicas y, por tanto, cambiantes.

- Las fases constructivas pueden seguirse sobre la fábrica mediante la identificación de líneas de rotura, diversidad técnica, cambio de materiales, huellas de talla, evolución de elementos ornamentales, etc.

- Cada una de las fases constructivas se encuentra en una posición estratigráfica relativa: es anterior, coetánea o posterior a otras. De no tener relaciones físicas directas, desconoceríamos esa posición.

-El establecimiento de cronologías absolutas debe apoyarse en técnicas multidisciplinares.
Pero aunque todos aceptemos los principios estratigráficos para realizar la lectura arqueológica de la arquitectura, siguen existiendo, a nivel teórico y de desarrollo epistemológico, cierta disparidad de criterios a la hora de fijar los criterios de profundización y documentación. Ya hemos señalado esta indefinición en otras ocasiones (LECANDA, 2000: 344) y hemos sido testigos de diversos debates tendentes a la normalización del proceso, pero si antaño fue por lo novedoso de la técnica y luego por su empleo selectivo y casi exclusivo en procesos imbuidos de un fuerte carácter investigador y dotados de todos los medios, más tarde por la extensificación de su empleo con escasa profesionalidad en muchos casos o por las limitaciones y condicionantes que le suelen acompañar $y$, finalmente por el manifiesto «choque» entre teoría y praxis cuando el método se somete en puridad metodológica a grandes arquitecturas históricas (véase, en este mismo simposio, AZKARATE, 2002: 65-67, sobre la Catedral de Santa María y su alegato por la simplificación de un registro que puede contener miles de unidades estratigráficas murarias), lo cierto es que parece necesario explicitar en cada ocasión los términos y conceptos empleados.

Entendemos que la acción mínima de análisis es la unidad estratigráfica muraria, que sirve para aislar y definir elementos constructivos y ofrece los fundamentos que nos permitirán establecer las cronologías relativas a través de una matriz de tipo Harris. Las relaciones de anteroposterioridad entre ellas (apoyar, cortar, adosar, rellenar, 
etc.) se visualizan en las juntas y uniones (CABALLERO y LATORRE, 1995).

La estratigrafía vertical debe analizar, como mínimo, las técnicas constructivas, los materiales empleados, los motivos decorativos, el acabado de los elementos, las fracturas, las dimensiones de huecos y vanos, las huellas de instrumental, los aglomerantes empleados y el espesor de las juntas (PARENTI, 1995).

Los análisis artísticos, químicos, documentales, etc. se desarrollan en paralelo pero independientemente; sus resultados se incorporan posteriormente en la matriz a fin de facilitar la interpretación histórica. Resultan muy útiles porque la estratigrafía define acciones constructivas pero no aporta interpretación artística y porque son éstos y no otros criterios los que sirven para establecer equivalencias entre las distintas acciones constructivas, operación que permite ordenar la secuencia y reconocer las Interfases de Periodo, o conjuntos de UU.EE.MM. que en un momento histórico concreto definen la forma del edificio. (BROGIOLO, 1995).

Nosotros hemos manejado en la catedral Vieja los siguientes niveles de análisis estratigráfico:

—Unidades Estratigráficas Murarias (UEM), que también pueden ser Interfaciales de Corte (UIC) o de Vano (UIV). En lo fundamental ya han sido definidas y responden a la conceptualización general de las mismas, pero en nuestra ficha de registro hemos simplificado algunos matices (p.e. UICorte vertical u horizontal), mientras que hemos añadido (como Parenti o Caballero) la UI de Vano, pues es uno de los pocos elementos que, por lo general, permiten una aproximación cronotipológica directa al edificio.

- Unidades Funcionales o Estructurales, conjunto de UU.EE.MM. definidas por su coherencia y unidad funcional y cronológica; una edificación es la suma de varias UF, que tienen en común el material empleado, la técnica constructiva, la funcionalidad, el ornamento, la cronología... El empleo de UF facilita el montaje de matrices, donde en casos como éste sería casi imposible reflejar la totalidad de las UEM.

-Fase Histórica o Interfase de Periodo, conjunto de UF que respondiendo a un mismo hiato cronológico suponen la fotografía sincrónica de un edificio en un momento histórico determinado. Es decir, definen la forma del edificio en cada una de sus etapas y, por tanto, nos muestra las características propias de cada uno de estos edificios.

\section{LA TOMA DE DATOS}

\section{La Documentación gráfica y planimétrica}

Se levantaron convencionalmente 10 planos, 8 plantas y 2 secciones, a escala 1:50, y se tomaron 70 fotoplanos recti- ficados digitalmente. Estas fotorrectificaciones no son exactamente fotogramétricas; por razones exclusivamente presupuestarias debieron ser sustituidas por una base fotográfica rectificada digitalmente realizada mediante un sistema denominado ELCOVISION 10 y que en lo sustantivo se diferencia de la fotogrametría terrestre en la ausencia de toma de datos topográficos y, derivado de ello, en la imposibilidad de ensamblar esos fotoplanos en un sistema de dibujo informático de tipo CAD. (SARTI y SARTI, 1997)

Con ellos se lograron recomponer 37 alzados, aunque de estos, 14 se realizaron con otros métodos complementarios, bien porque resultaba imposible disparar las cámaras a tan corta distancia, bien porque las limitaciones presupuestarias no permitieron más tomas.

\section{El análisis documental}

Realizado bajo la responsabilidad del Dr. Martín Viso, presentó dos fases distintas; en primer lugar la de expurgo documental y bibliográfico de lo publicado hasta la fecha y, después, la búsqueda de nuevas informaciones sobre la historia del edificio en sus propios archivos (Archivo Catedralicio). No creemos necesario adentrarnos en los principios metodológicos de una ciencia como la Historia. Sus resultados fueron plasmados en un estudio monográfico, inédito (MARTín VIso, 2000) e independiente pero anexo a nuestro trabajo, y donde tal vez lo más novedoso, amén de un par de documentos nuevos relativos a ciertas obras en la Catedral Vieja, sea que se procura un acercamiento histórico al edificio no sólo a través del puro documento, sino también del contexto histórico en el que éste se enmarca.

\section{La diferenciación de UU.EE.MM}

Para su determinación se emplearon los criterios discriminatorios habituales según la propuesta de Parenti, contemplando su registro en una ficha normalizada, como las que pueden verse en la bibliografía (PARENTI, 1995 y 1996) pero con ligeras adaptaciones. En éstas tienen un mayor peso específico los campos destinados al registro descriptivo, quedando los aspectos interpretativos ahora muy reducidos, pues no es este el momento de su determinación (lo será en la cuarta fase del programa). En la redacción de la ficha se procura describir las acciones constructivas tanto desde el punto de vista arqueológico como desde el arquitectónico, lo que en este segundo caso añade cierto valor interpretativo (funcional cuando menos).

Se han obtenido unas 300 fichas de UU.EE.MM., que fueron identificadas con un sistema alfanumérico, común para todos los ámbitos de estudio pero que permitía, al mismo tiempo, una diferenciación de cada uno de esos contextos. El sistema, propuesto por Caballero (1995), 
consistió en la asignación de series numéricas independientes para cada ámbito (p.e. Mazmorra, 1001-1100, Esc. Tramos Rectos, 1101-1200, etc.), lo que da autonomía a cada espacio para desarrollarse cuanto necesite (aunque se previó que las series no pasarían de 100 unidades). Su asignación, absolutamente arbitraria, siguió la lógica de los recorridos y la existencia de puntos de conexión estratigráfica entre los distintos ámbitos.

\section{Secuencia Constructiva Relativa}

El resultado final de la toma de datos tiene que ser una matriz de tipo Harris, donde se deje constancia de la secuencia cronológica — constructiva - relativa. El diagrama podría elaborarse a partir de las UU.EE.MM. individualizadas, aunque ello resulta difícil por su número, su pertenencia a espacios muy compartimentados y distantes y por sus relaciones redundantes, de ahí que hayamos considerado más conveniente hacerlo por medio de su simplificación en Unidades Funcionales.

En este diagrama de periodización relativa podrá irse incorporando la información absoluta que de las analíticas encargadas se obtengan, dotando a la secuencia de cronologías absolutas.

\section{El sondeo arqueológico}

En un punto muy concreto de nuestra zona de estudio se consideró oportuno, ante las demandas de la administración y de los técnicos en edificación, la realización de un pequeño sondeo arqueológico a fin de verificar la existencia de suelos previos, de rellenos arqueológicos, de cotas de cimentación y otros temas similares. Las posibilidades que $a$ priori ofrecía ese espacio nos parecían mínimas, sobre todo por lo reducido y limitado de la intervención, pero nuestro trabajo, en éste caso, debe recordarse, está al servicio de la restauración y de sus necesidades.

El sondeo se practicó en la zona del Acceso Viejo a Torre Mocha, un acceso descubierto durante las obras de restauración y donde se localiza el paramento que parece ser el más antiguo de la secuencia.

La excavación, bajo la dirección de quien suscribe, fue ejecutada por Aratikos Arqueólogos S.L. y los resultados, como cabría esperar, fueron insignificantes a los efectos de este trabajo.

\section{Análisis realizados y toma de muestras para la Segunda Fase}

Durante la fase de toma de datos y lectura estratigráfica se practicaron algunos análisis encaminados a la más precisa determinación de características materiales de algunas de las UU.EE.MM. documentadas e, indirectamente, de algu-

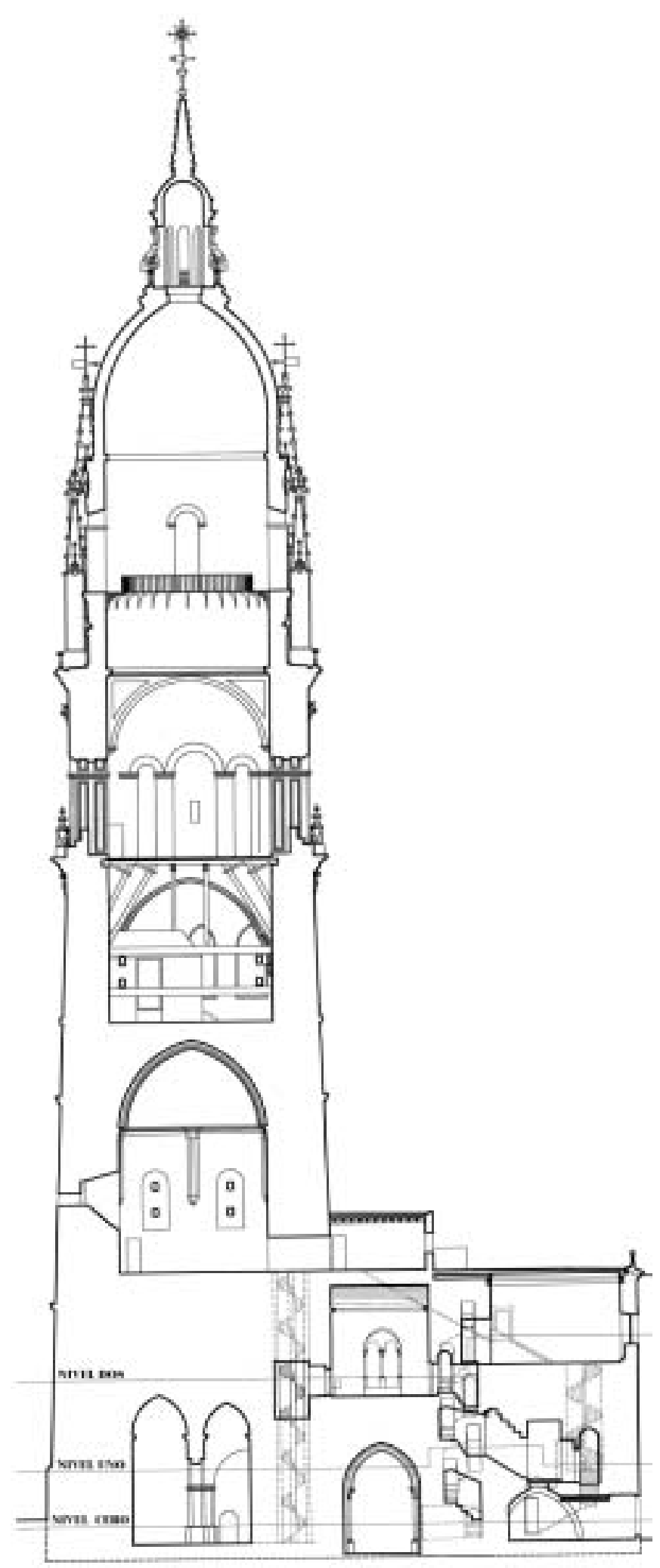

Fig. 1. Sección del área de estudio: Cuerpo de Torres de la Catedral Vieja 
nas características técnicas o materiales de algunas de las fases históricas. Fueron de dos tipos, uno geológico y otro metalográfico; el primero para estudiar y catalogar el material lítico constructivo y verificar si existen diferencias en la piedra empleada durante las distintas fases constructivas; el segundo, por ver si a través del análisis del material metálico empleado en la construcción (grapas, clavos, etc.) podemos llegar a precisiones cronológicas derivadas de la técnica y calidad de su forja. Ambos se llevaron a cabo por parte de especialistas de la Univ. Complutense de Madrid.

Pero además, y en previsión de los requerimientos dimanantes de la anunciada Segunda Fase (aún no acometida), durante nuestro trabajo se fueron tomando una serie de muestras de distinta naturaleza (ladrillo, madera, argamasa, etc.) en previsión de futuros análisis: de datación, de composición, pruebas mecánicas del material constructivo, etc.

Al final solo se nos permitió remitir a los correspondientes laboratorios las muestras destinadas al carbono 14 (Univ. de Granada y Univ. de Uppsala, Suecia) y a la termoluminiscencia (Unv. Autónoma de Madrid).

Sobre todos estos extremos puede obtenerse mayor información en el correspondiente informe de intervención, depositado en la Junta de Castilla y León (LECANDA, 2000 b).

\section{CONCLUSIONES PROVISIONALES DE LA PRIMERA FASE DEL ANÁLISIS ESTRATIGRÁFICO DEL CUERPO DE TORRES DE LA CATEDRAL VIEJA DE SALAMANCA}

Como habrá podido comprender el lector, poco es, en términos de narración histórica, lo que ahora podemos decir acerca de la historia constructiva del Cuerpo de Torres de la Catedral Vieja, pues como a lo largo de estas páginas hemos intentado explicar nuestro trabajo sólo ha consistido, hasta la fecha, en la toma de datos para la lectura estratigráfica del edificio.

Bien es cierto que aunque nunca se ha superado oficialmente esa fase sí dimos los primeros pasos de la siguiente, la realización de analíticas encaminadas, básicamente, a la obtención de dataciones absolutas.

Éstas, dispuestas sobre la matriz y unidas a la identificación de las distintas Unidades Estructurales e inmersas en el conocimiento histórico procedente del estudio documental, constituirán el esqueleto argumental de nuestra interpretación histórica y, al tiempo, configurarán el soporte probatorio de tal evolución constructiva.

En breves líneas adelantaremos los aspectos mas significativos de nuestras conclusiones, aunque éstas, como de su naturaleza y origen cabe esperar, no superen el grado de provisionales, pero con base empírica.
En la construcción del Cuerpo de Torres de la Catedral Vieja de Salamanca se han documentado siete (7) Fases Constructivas, a saber: dos románicas (siglos XII y XIII respectivamente), dos góticas (una del XIV/XV y otra ya cercana al XVI), dos barrocas (del XVII y XVIII respectivamente) y una contemporánea y reciente.

Inicialmente no parece detectarse la construcción sobre restos arquitectónicos previos; por el contrario, los primeros testigos constructivos parecen denunciar el replanteo e inicio constructivo de un edificio de tipología románica, con dos torres en el muro hastial, donde se localiza la fachada principal, sobre un solar vacío o vaciado al efecto y del que se reutilizan materiales, incluso romano imperiales, para la nueva construcción. Todo parece indicar que los comienzos de la obra deben situarse en los albores del siglo XII.

Esa fase se refleja en diversas UU.EE.MM. del Acceso Viejo, Escalera Vieja en sus dos tramos, Husillo Cegado y Capilla de San Martín.

Razones diversas provocan una rectificación del replanteo inicial, así como un cambio en los gustos estéticos (lo que ya había detectado la Historia del Arte), lo que provoca la diferenciación de una nueva fase constructiva, estilísticamente románica de tipo zamorano y datable en la parte inicial del siglo XIII.

En el terreno del arte remitimos al lector a las obras de Lampérez (1908) y de Yarza (1985).

Muestras de esta fase se documentan en Mazmorra, Escalera de Tramos Rectos Mazmorra-carcelero, Carcelero, terraza de Torre Mocha, Acceso Viejo, Escalera Vieja, Alcaide y Husillo Cegado.

La marcha de las obras y los nuevos planteamientos se prolongan a lo largo del siglo XIV, en una fase estilísticamente ya gótica aunque de alcance bastante local, registrándose evidencia de estos trabajos en Sala del Alcaide (conformada en nuestra opinión en estos momentos a modo de balconada o tribuna exterior entre las dos torres de la fachada) y en el Husillo Cegado.

Este mismo espacio articulado en torno a la sala del Alcaide es el que más se ve afectado por las obras góticas del XVI, causadas, más que por un interés constructivo específico en este sector, como consecuencia del replanteo e inicio de la las obras de la Catedral Nueva, que, como sabemos, se inició, curiosamente, por los pies.

Las dos fases barrocas, del XVII y del XVIII, afectan a prácticamente toda la masa edificatoria del Cuerpo de Torres, aunque con una diferencia sustantiva: las primeras son de origen estético, mientras que las segundas están causadas por la pura necesidad de mantener en pie la Catedral.

En las primeras, por ejemplo, se cierra la tribuna que era la Sala del Alcaide, se abren nuevos vanos bajo arcos re- 
bajados en las fachadas o se cierran y cambian otros hacia el interior del templo, a la vez que se embellecen, según criterios estéticos propios del momento, las fachadas exteriores.

Hay un dato arqueológico que caracteriza bastante bien, en nuestra opinión, esta fase del XVII, en la que se procura seguir empleando la misma arenisca hasta entonces utilizada en la construcción, pero o bien la fuente debió variarse ligeramente por agotamiento de las vetas primigenias, o bien no existían los recursos necesarios como para permitirse el descartar ciertos bloques con visibles clastos de concreciones carbonatadas.

En las obras de conservación y mantenimiento del siglo XVIII, por los daños sufridos en las torres con motivo de diversas causas y, en última instancia, por el terremoto de Lisboa, se forran los paramentos exteriores para darles mayor solidez, se ciegan los husillos a base de cal y canto convirtiéndolos en soportes estructurales de las torres, se compartimenta y varía el sentido y uso de los espacios iniciales, se modifica la terraza por un espacio bajo cubierta, etc., siendo éstas situaciones y acciones señaladas expresamente en diversas fuentes escritas.

Una muestra evidente de cómo el sentido de las intervenciones ha cambiado entre una y otra fase barroca puede observarse con claridad en la fachada, sobre la puerta de acceso, donde se sitúa la Sala del Alcaide. En el primer barroco se cierra ese espacio con un muro presidido exteriormente por un conjunto escultórico mariano bajo arcosolio y por una serie de óculos que articulan la fachada; toda la simetría compositiva buscada se pierde cuando en el segundo barroco se hace necesario reforzar la Torre de Campanas con un nuevo paramento exterior ataluzado; entonces, éste, remonta parcialmente la fachada central amortizando parte de la misma y "desplazando" el eje de simetría, con lo que se pierde el sentido compositivo original.

Por lo que respecta a las evidencias correspondientes a la última fase constructiva, la contemporánea, básicamente reciente y vinculadas a trabajos de restauración en el edificio, no creemos necesario mayor comentario.

\section{Bibliografía}

AZKARATE A, 2002, Intereses cognoscitivos y praxis social en Arqueología de la Arquitectura, Arqueologia de la Arquitectura, 1, pp.55-71.

BerRiochoA V., 1997-98, Plan director de la Catedral de Salamanca, Ars Sacra, 4-5, pp. 113-126.

BerriochoA V., 1997, Plan Director de la Catedral de Salamanca. II Fase. Inédito, Servicio Territorial de Cultura de Salamanca. Junta de Castilla y León.

BERRIOCHOA V., 1997, Proyecto de Restauración interior de Torre Mocha y Torre deCampanas de la Catedral de Salamanca. Inédito. Servicio Territorial de Cultura de Salamanca. Junta de Castilla y León.

BROGiolo G.P., 1995, Arqueología estratigráfica y restauración, Informes de la Construcción, 435, pp.31-36.

Caballero Zoreda L., 1995, Método para el análisis estratigráfico de construcciones históricas o «lectura de paramentos», Informes de la Construcción, 435, pp.37-46.

Caballero L., RetUerCe M., 1998, Excavación arqueológica en el patio de la Catedral de Salamanca. Inédito. Servicio Territorial de Cultura de Salamanca.

CAmón AZnar J., 1958, Las etapas de la Catedral Vieja de Salamanca, Goya, XXIII, pp. 274-280.

GONZÁLEZ J.,1943, La Catedral Vieja de Salamanca y el probable autor de la Torre del Gallo, Archivo Español de Arte, XV, pp. 39-50.

LAmpérez Y RomeA V., 1908, Historia de la Arquitectura Cristiana Española en la Edad Media. Tomo 1, Madrid.

LATORRE P. Y CABALLERo L., 1995, La importancia del análisis estratigráfico de las construcciones históricas en el debate sobre la restauración monumental, Informes de la construcción, n. ${ }^{\circ} 435$, pp. 5-18.

LECANDA J.A., 1998 (e.p.) El estudio arqueológico y la restauración de San Salvador de Escaño (Merindad de Castilla La Vieja), Burgos, Actas de las VII Jornadas sobre Rehabilitación de Edificaciones Antiguas. Almendralejo (abril de 1998).

LECANDA J.A., 2000, El análisis arqueológico de la iglesia de San Salvador de Escaño: resolución de problemas arquitectónicos y aproximación histórica al primer románico burgalés, Actas del V Congreso de Arqueología Medieval Española, Valladolid, Vol.1, pp. 341-349.

LECANDA J.A., 2000 b, Análisis estratigráfico para la restauración de la Tore Mocha y Torre de Campanas (Catedral de Salamanca): Toma de datos y registro arqueológico. Inédito. Servicio Territorial de Cultura de Salamanca. Junta de Castilla y León.

MarTín VIso I., 2000, Análisis estratigráfico para la restauración de Torre Mocha y Torre de Campanas de la Catedral de Salamanca: Estudio documental y de contexto histórico. Inédito. Servicio Territorial de Cultura de Salamanca. Junta de Castilla y León.

PARENTI R., 1995, Historia, importancia y aplicaciones del método de lectura de Paramentos, Informes de la construcción, n. ${ }^{\circ} 435$, pp. 19-30.

PARENTI R., 1996, Individualización de las unidades estratigráficas murarias, en Caballero L., Escribano C. (eds.), 1996, Actas. Arqueología de la Arquitectura, Salamanca, pp. 75-86.

PORTal Monge M. R.Y., 1992, Sobre la construcción de Santa María de la Sede o Catedral Vieja de Salamanca: siglos XII-XIV. Salamanca: revista de estudios, 29-30, pp. 75-94.

PorTal Monge M.R.Y., 1997, Noticias documentales sobre las obras en Santa María de la Sede o Catedral Vieja de Salamanca durante los siglos XVI-XX. Salamanca: revista de estudios, 29-30, pp. 445-457.

Sarti Fernández M.A., SARTI FernándeZ F., 1997, Levantamiento de planos en la restauración, $R \mho R$ Restauración y Rehabilitación, 3, pp. 86-89.

YARZA J., 1985 (5.a), Arte y arquitectura en España, 500-1250, Madrid. 